Sousa ljO, Pereira RnS, Roseane Pedrosa lima, Rosa GS, GONÇALVES RLG, NETO MPL
ANÁLISE DA TOXICIDADE DO EXTRATO HIDROALCOÓLICO DA POUPA DO FRUTO SECO DE LUFFA OPERCULATA FRENTE A NÁUPLIOS DE ARTEMIA SALINA E MERISTEMAS DE ALLIUM CEPA

\title{
ANÁLISE DA TOXICIDADE DO EXTRATO HIDROALCOÓLICO DA POUPA DO FRUTO SECO DE LUFFA OPERCULATA FRENTE A NÁUPLIOS DE ARTEMIA SALINA E MERISTEMAS DE ALLIUM CEPA
}

Analysis of the toxicity of the hydroalcoolic extract of the dry fruit pulp of Luffa operculata in metodology Artemia salina and Allium cepa

Análisis de la toxicidad del extracto hidroalcohólico de la pulpa del fruto seco de Luffa operculata frente al náuplios de Artemia salina y meristemas de Allium cepa

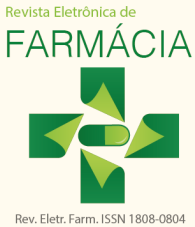
Ian Jhemes Oliveira Sousa1*, Raimunda Nonata dos Santos Pereira², Roseane Pedrosa Lima², Gardenha da Silva Rosa $^{3}$, Rodrigo Lopes Gomes Gonçalves ${ }^{1,3}$, Manoel Pinheiro Lúcio Neto ${ }^{1,2,4}$

${ }^{1}$ Universidade Federal do Piauí (UFPI)

2 Instituto de Educação Superior de Teresina (IEST/AESPI)

${ }^{3}$ Centro de Ensino Tecnológico de Teresina (CET)

${ }^{4}$ Faculdade Santo Agostinho (FSA)

*E-mail: ianjhemes@gmail.com

\section{RESUMO}

Introdução: Este trabalho avaliou a toxicidade do extrato bruto hidroalcoólico de Luffa operculata, para tanto foram executados ensaios de toxicidade in vitro com Allium cepa e Artemia salina.

Objetivo: A espécie foi estudada por ser uma importante planta medicinal para os nordestinos no tratamento de problemas respiratórios e devido à carência de literatura com essa abordagem; dessa forma o objetivo desse trabalho é estabelecer limites toxicológicos para esta planta utilizando metodologias de quantificação toxicológica in vitro.

Métodos: O extrato foi preparado por meio de decocção hidroalcoólica seguido de rotaevaporação, da avaliação da toxicidade frente a viabilidade de náuplios de Artemia salina e do crescimento de meristemas de Allium cepa.

Resultados: Os resultados mostraram que a concentração letal 50\% $\left(\mathrm{CL}_{50}\right)$ foi: 25,22 e 4,63 $\mu \mathrm{g} / \mathrm{mL}$ para 24 e 48 horas respectivamente, para a metodologia de Artemia salina e a inibição do crescimento meristemático de Allium cepa foi significante a partir de $500 \mu \mathrm{g} / \mathrm{mL}$.

Conclusão: Estes resultados constatam as hipóteses da alta toxicidade dos compostos da Luffa operculata, além de seu possível potencial citotóxico com concentrações acima de $500 \mu \mathrm{g} / \mathrm{mL}$ estabelecendo um perfil toxicológico para mais estudos detalhados da toxicologia desta espécie.

Palavras-chave: Cucurbitaceae. Plantas medicinais. Toxicologia.

\section{ABSTRACT}

Introduction: This study evaluates the toxicity of the crude hydroalcoholic extract of Luffa operculata for both in vitro toxicity tests: Allium cepa and Artemia salina. The species in question was studied because it is an important medicinal plant for the Northeastern. Besides that, this specie is utilized in the treatment of respiratory problems and there is a shortage of literature on the toxicological safety of this species.

Objective: Therefore, the objective of this work is to establish toxicological limits for this plant using in vitro toxicological quantification methodologies.

Methods: The extract was prepared by means of hydroalcoholic decoction followed by rotavaporation, toxicity evaluation against the viability of Artemia salina nauplii and growth of Allium cepa meristem. 
Results: The results showed that the $L_{50}$ was: 25.22 and $4.63 \mu \mathrm{g} / \mathrm{mL}$ for 24 and 48 hours, respectively for the Artemia saline methodology. The inhibition of the meristematic growth of Allium cepa was significant from $500 \mu \mathrm{g} / \mathrm{mL}$.

Conclusion: These results confirm the hypothesis of the high toxicity of Luffa operculata compounds, in addition to their potential cytotoxic with concentrations above $500 \mu \mathrm{g} / \mathrm{mL}$, establishing a toxicological profile for further detailed studies of the toxicology of this species.

Keywords: Cucurbitaceae. Medicinal Plants. Toxicology.

\section{RESUMEN}

Introducción: Este trabajo evalúo la toxicidad del extracto bruto hidroalcohólico de Luffa operculata. Para eso, fueron realizados experimentos de toxicidad in vitro con Allium cepa y Artemia salina. Objetivo: La especie fue estudiada por ser una planta medicinal importante para la población nordestina de Brasil en el tratamiento de problemas respiratorios y por causa de la falta de literaria sobre este tema. De esta manera, el objetivo de este trabajo experimental es establecer límites toxicológicos para esa planta utilizando metodologías de cuantificación toxicológicas in vitro.

Métodos: El extracto fue preparado a través de decocción hidroalcohólica, seguido de rotaevaporación, la evaluación de la toxicidad frente a la viabilidad de náuplios de Artemia salina y crecimiento de meristemas de Allium cepa.

Resultado: Los resultados mostraron que la concentración letal 50\% (CL50) fue: 25,22 y 4,63 ug/mL para 24 y 48 horas respectivamente, para la metodología de Artemia salina y la inhibición del crecimiento meristemático de Allium cepa fue significante a partir de $500 \mathrm{ug} / \mathrm{mL}$.

Conclusion: Esos resultados constatan las hipótesis de la alta toxicidad de los compuestos de la Luffa operculata, además de un posible potencial citotóxico con concentraciones mayor que $500 \mathrm{ug} / \mathrm{ml}$, estableciendo un perfil toxicológico para más estudios detallados de la toxicología de esta especie.

Palabras clave: Cucurbitaceae. Plantas Medicinales. Toxicología.

\section{INTRODUÇÃO}

O uso de plantas na terapêutica é tão antigo quanto a própria espécie humana. Luffa operculata Cogn. é uma angiosperma nativa da América do Sul, em especial do Brasil, e trata-se de uma dicotiledônea que pertence à família Cucurbitaceae, a qual é conhecida popularmente como buchinha, cabacinha, purga-de-jalapa, purga-dos-paulistas e etc ${ }^{(1)}$. Esta planta vem sendo empregada na medicina popular tradicional, especialmente para transtornos do trato respiratório superior como sinusite, rinite e até como descongestionante nasa ${ }^{(2,3)}$. O fruto seco da Luffa operculata é vastamente utilizado como fitoterápico, apresentando diversas indicações, formulações e apresentações. Compostos a base desta planta são geralmente encontrados em mercados, farmácias de manipulação, bancas de ambulantes e vendedores de ervas medicinais nas mais diversas regiões do Brasil, é muito difundido no interior do país com o preparado conhecido como "garrafada", um extrato aquoso da planta em conjunto com outras ervas, utilizada como abortiva e purgativa, demonstrando sua ação como poderoso irritante de mucosas ${ }^{(2)}$.

Dentro da realidade de utilização de produtos naturais, é importante salientar que fitoterápicos podem provocar reações adversas, sendo que essas reações podem ocorrer por ação direta de seus próprios constituintes, devido a interações com outros medicamentos ou alimentos, ou até mesmo reações adversas causadas por características do paciente (idade, condições fisiológicas, fator genético, entre outros)(4).

Dentro do contexto no qual se busca verificar o potencial toxicológico de plantas medicinais com diversos métodos confiáveis e economicamente acessíveis, se mostram promissores para ensaios toxicológicos preliminares que permitem quantificar uma relação quantitativa do composto e sua toxicidade, informação essa que pode contribuir para o estudo de toxicidade de plantas medicinais ${ }^{(3)}$. 
Muitos laboratórios de Produtos Naturais têm inserido dentro de suas rotinas de diversos ensaios biológicos simples como a metodologia de toxicidade quantitativa com a utilização do crustáceo Artemia salina L., que é proposto como um bioensaio relativamente simples para avaliar atividades farmacológicas e toxicológicas de produtos naturais. Através desse método é possível determinar a concentração letal $50 \%\left(\mathrm{CL}_{50}\right)$ de substâncias e extratos de origem vegetal ou animal em um meio salino. O princípio do teste é a manifestação de toxicidade de componentes ativos, frações ou extrato de produtos naturais frente ao organismo marinho Artemia salina através da sua morte ou imobilização permanente no qual esse simples organismo pode ser usado como um monitor conveniente para a toxicidade de produtos, além de ser um método rápido, seguro e acessível(5).

A Artemia salina é considerada uma espécie de micro crustáceo bivalve de água salgada que geralmente é utilizada como alimento vivo para peixes, os quais seus cistos são facilmente adquiridos em lojas aquaristas. Na literatura diversos trabalhos se propõem a correlacionar os parâmetros de toxicidade em Artemia salina com atividades farmacológicas, tais como antifúngica, viruscida e antimicrobiana, parasiticida, tripanossomicida, entre outras. ${ }^{(5,6)}$

Para análise citotóxica preliminar in vitro de compostos biológicos, o teste com Allium cepa é uma abordagem com grande potencial, pois o uso de raízes de Allium cepa possibilita avaliar variações de $\mathrm{pH}$ e a presença de resíduos metálicos como chumbo, níquel cromo, zinco, cobre e cádmio, permitindo avaliação rápida e segura do ponto de vista de confiabilidade, revelando o efeito danoso das substancias DNA em que culmina a cinética de divisão celular ${ }^{(4,6)}$. Além disso, uma série de estudos corroboram com a utilização do teste de Allium cepa como um ensaio importante na avaliação de extratos e infusões de plantas medicinais ${ }^{(7,8)}$.

O objetivo deste estudo é quantificar a toxicidade do extrato hidroalcoólico (EHA) da poupa do fruto seco de Luffa operculata frente a exposição a náuplios de Artemia salina e a avaliação frente a inibição do crescimento meristemático de Allium cepa.

\section{METODOLOGIA}

\section{Obtenção do material vegetal}

O fruto (baga) seco da Luffa operculatta foi coletado no interior da cidade de Joselândia, que se localiza no seguinte ponto de coordenadas: $04^{\circ} 58^{\prime}$ Sul e $44^{\circ} 42^{\prime}$ Oeste, parte do estado do Maranhão - Brasil, situando-se a $60 \mathrm{~m}$ acima do nível do mar. A coleta dos frutos parcialmente secos foi realizada entre $9 \mathrm{~h}$ e $11 \mathrm{~h}$ da manhã, e posteriormente, foram secos à sombra por 3 dias. Uma amostra da espécie foi coletada para a confecção da exsicata e identificação da espécie, que posteriormente foi depositada no Herbário Graziela Barroso da Universidade Federal do Piauí - UFPI, recebendo o número de registro TEPB30.939 (Luffa operculata Cogn.).

\section{Preparação dos Extratos}

As bagas secas foram cortadas para a remoção das sementes e processadas por moinho de facas até atingir um nível de fragmentos pequenos o suficiente para a extração. O material utilizado para extração (porção de 50 gramas) foi levado ao Laboratório Interdisciplinar da Universidade Federal do Piauí - UFPI, vinculado ao curso de Farmácia. Foi pesado em balança semianalítica 50g da popa do fruto seco sem as sementes. O material foi colocado em béquer e submetido à decocção em uma solução hidroalcoólica a 70\% (400 ml de Álcool etílico Absoluto (Vetec Q. F.) e $170 \mathrm{ml}$ de água destilada) por um período de 10 minutos, após atingir a ebulição em temperatura de $75 \pm 5$ graus Celsius. Após isso, submeteu-se o material a filtração e o filtrado foi depositado no balão do rotaevaporador previamente pesado $(274,46 \mathrm{~g})$. O processo de secagem a vácuo no rotaevaporador $\left(50^{\circ} \mathrm{C}\right.$ e pressão negativa de -700 $\mathrm{mmHg} \pm-100 \mathrm{mmHg}$ e $50 \mathrm{rpm}$ ) durou cerca de $4 \mathrm{~h} 30 \mathrm{~min}$. Em sequência, pesou-se o balão contendo o extrato seco após resfriamento a temperatura ambiente, conferindo $281,22 \mathrm{~g}$, e, consequentemente, $6,76 \mathrm{~g}$ do extrato (13,52\% de rendimento do processo). 


\section{Avaliação da toxicidade aguda frente a náuplios Artemia salina}

Para o experimento foram utilizados cistos de Artemia salina que foram adquiridos em loja especializada em produtos para aquário e levados ao Laboratório Interdisciplinar I do Centro de Ensino Tecnológico de Teresina. Para obtenção das condições desejadas à eclosão dos cistos de Artemia salina, a água do mar artificial foi preparada com seguintes componentes: água mineral natural, bicarbonato de sódio (Dinâmica Q.C.) (5 g/L) e cloreto de sódio (Dinâmica Q.C.) (30 g/L).

A solução de água do mar artificial foi previamente aerada durante 30 minutos e então realizada a incubação de 100 mg de cistos de Artemia salina por 48 horas em béquer, onde durante esse período os cistos foram mantidos em iluminação de 10 W (lâmpada de led), sendo realizado o controle de temperatura para manter a variação máxima entre 25 a $30^{\circ} \mathrm{C}$.

O bioensaio de toxicidade frente à Artemia salina foi realizado segundo a metodologia proposta por Meyer ${ }^{(9)}$ com algumas adaptações, as quais os náuplios foram transferidos em quantidade de 10 indivíduos para cada tubo de ensaio, sendo o volume completado para 4,9 mL da solução salina previamente preparada (água do mar artificial) e posteriormente adicionado $100 \mu \mu l$ do extrato hidroalcoólico seco (EHA) diluído em solução de dimetilsulfóxido (Synth Q.F.) a 25\% (DMSO) em diferentes concentrações $(1,25 ; 2,5 ; 5 ; 12,5 ; 25 ; 50$ e $100 \mathrm{mg} / \mathrm{mL})$ para cada tubo, de forma que cada um ficasse com as respectivas concentrações (25; 50; 100; 250; 500; 1000 e $2000 \mu \mathrm{g} / \mathrm{mL}$ ). Todas as concentrações foram realizadas em triplicatas. Para o controle negativo foi utilizado apenas a solução salina + DMSO, e foi aproveitado o mesmo esquema de diluição de concentrações, o dicromato de potássio $\left(\mathrm{K}_{2} \mathrm{Cr}_{2} \mathrm{O}_{7}\right)$ (Synth Q.F.) foi utilizado como padrão de avaliação de toxicidade.

Os náuplios de Artemia salina ficaram sob exposição por 24 e 48 horas sob iluminação artificial (10 W). Decorrido esse período de contato, os sobreviventes foram contados com o auxílio de lupas, na análise foram consideradas larvas mortas todas que não apresentavam qualquer movimento ativo em cerca de dez segundos de observação.

Figura 1. Esquematização da metodologia de avaliação toxicológica com náuplios de Artemia salina

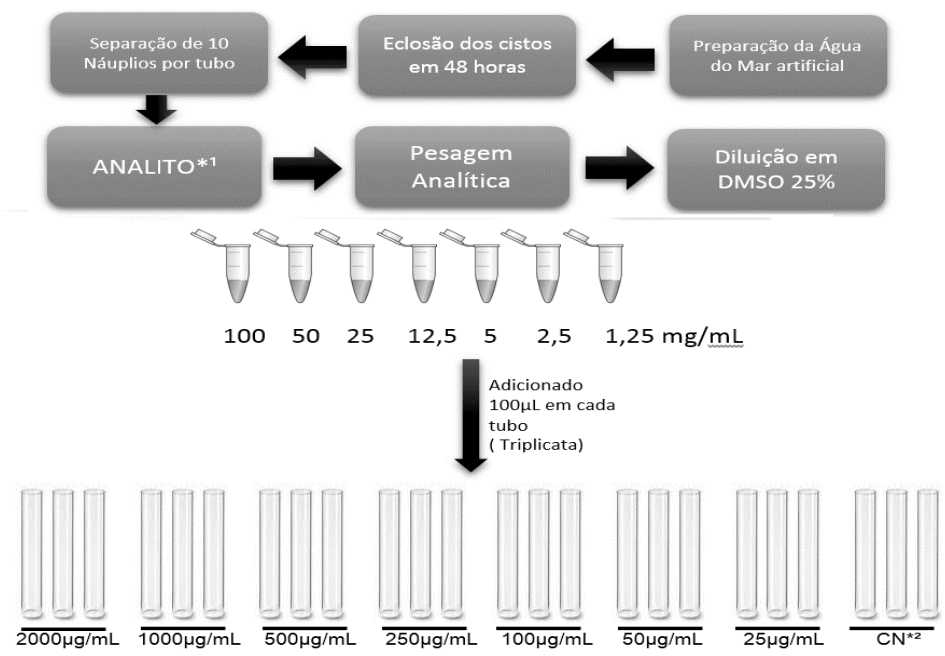

Legenda: *1 Foram executados dois ensaios independentes com as mesmas concentrações para os respectivos: Extrato Bruto Hidroalcoólico (EBHA) de Luffa operculata e para o Dicromato de potássio . *2 O controle negativo utilizado foi a água do mar sintética + DMSO.

Fonte: Elaborado pelo autor lan Jhemes Oliveira Sousa (2017). 
Avaliação da inibição do crescimento de meristemas de Allium cepa L.

A metodologia utilizada no teste de Allium cepa recorre-se ao protocolo sugerido primeiramente por Fiskesjö(10), com modificações por Sturbelle ${ }^{(11)}$. Os tratamentos foram realizados utilizando uma quantidade total de cinco bulbos de Allium cepa (quintuplicata) para cada concentração da substância a ser testada, os quais foram selecionados e padronizados por lavagem em água corrente por cerca de 30 minutos. As raízes foram cultivadas em condições controladas de temperatura (25 $\pm 5^{\circ} \mathrm{C}$ ) em recipientes inertes contendo os extratos nas concentrações $(25 ; 50 ; 100 ; 250 ; 500 ; 1000$ e $2000 \mu \mathrm{g} / \mathrm{mL}$ ) diluídos em água mineral, ficando incubados durante 72 horas com reposição de solução a cada intervalo de 12 horas. Para controle negativo utilizou-se água mineral, o paracetamol (Biosintética Farm.) 1,0 mg/mL foi utilizado como controle positivo. A avaliação consistiu em medição e comparação estatísticas de crescimento das raízes dos grupos de bulbos cultivados em contato com as concentrações do EBHA de Luffa operculata.

\section{Análise Estatística}

Os resultados foram avaliados por meio da análise de "Sigmoidal Dose-Resposta" (Artemia salina) e Análise de Variância (ANOVA) seguido por teste multiparamétrico de Tukey (Allium cepa) utilizando o software Graphpad Prism 5.0 (Intuitive Software for Science, San Diego, C.A.) considerando $p<0,05$.

\section{RESULTADOS}

Os resultados obtidos a partir da análise da toxicidade da Luffa operculata frente a náuplios de Artemia salina estão dispostos nas Figuras 2 e 3 respectivamente, para as análises do comportamento dos náuplios frente a exposição em 24 e 48 horas ao EBHA de Luffa operculata.

Figura 2. Relação de viabilidade dos náuplios frente as concentrações seriadas de Luffa operculata e de Dicromato de potássio por 24 horas

\section{Toxicidade aguda do extrato de L. operculata em 24 horas versus Dicromato de potássio}

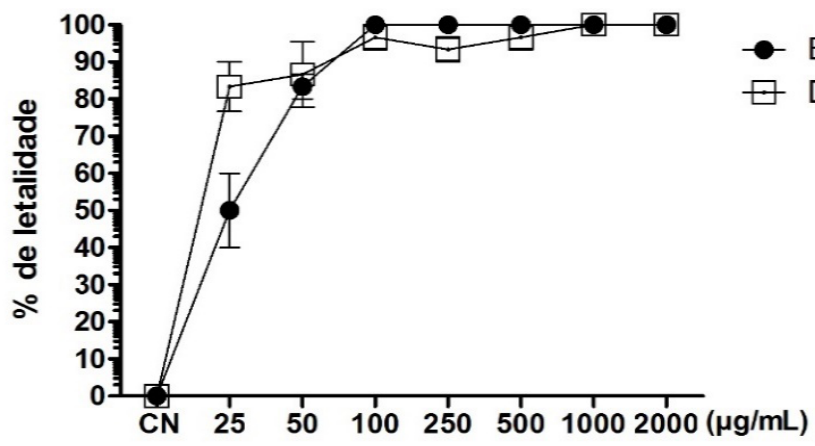

Legenda: Os resultados estão expressos como Média Erro Padrão Médio e água do mar sintética + DMSO foram utilizados como Controle Negativo (CN). Fonte: Elaborado pelo autor lan Jhemes Oliveira Sousa (2017). 
A comparação da toxicidade do Extrato Bruto Hidroalcoólico de Luffa operculata frente ao dicromato de potássio mostrou que há um aumento na concentração dose-dependente havendo, que chega ao platô (100\% de letalidade) a partir de $100 \mu \mathrm{g} /$ $\mathrm{mL}$, no qual até a concentração de $50 \mu \mathrm{g} / \mathrm{mL}$ o EBHA apresenta-se levemente menos tóxico que dicromato de potássio, onde acima disso a toxicidade de ambos torna-se similar.

Figura 3. Viabilidade dos náuplios frente as concentrações seriadas de Luffa operculata e de Dicromato de potássio por 48 horas

\section{Toxicidade aguda do extrato de $L$. operculata em 48 horas versus Dicromato de potássio}

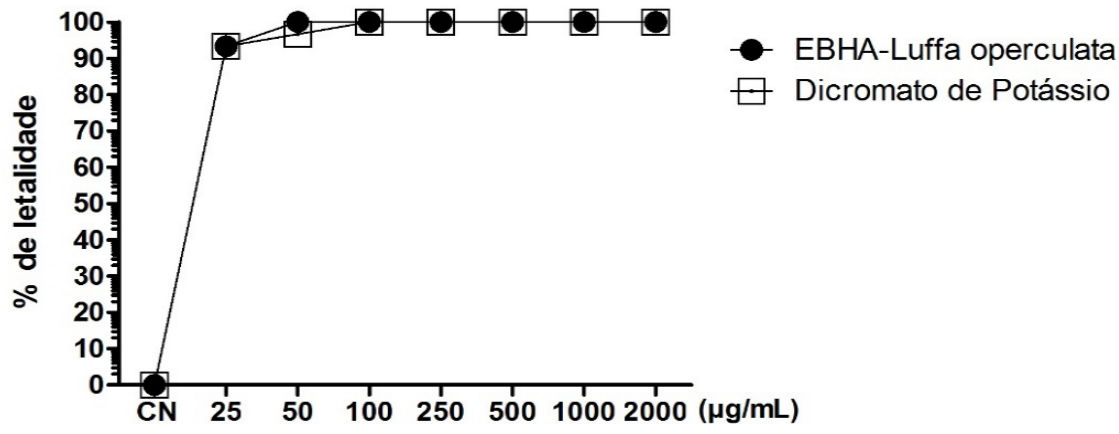

Legenda: Os resultados estão expressos como Média Erro Padrão Médio e água do mar sintética + DMSO foram utilizados como Controle Negativo (CN). Fonte: Elaborado pelo autor lan Jhemes Oliveira Sousa (2017)

Os resultados obtidos na avaliação da viabilidade dos náuplios de Artemia salina para 48 horas mostram que em exposição prolongada até mesmo as concentrações mais baixas da ordem de $25 \mu \mathrm{g} / \mathrm{mL}$ são altamente tóxicas aos náuplios, representando que até mesmo em concentrações muito baixas (25 partes por milhão) o extrato mostra-se com elevada toxicidade.

Para uma observação melhor da avaliação da toxicidade frente a Artemia salina, os resultados foram avaliados por meio de uma análise estatística de regressão linear para obter os valores de $\mathrm{CL}_{50}$, para assim melhor definir os limites toxicológicos do extrato desta espécie.

Tabela 1. Cálculos estatísticos da "Sigmoidal dose-resposta" realizados para o cálculo da $\mathrm{CL}_{50}$

\begin{tabular}{|lll|}
\hline PARÂMETRO & $\mathbf{2 4}$ Horas & $\mathbf{4 8}$ Horas \\
\hline EC $_{\mathbf{5 0}}$ & $25,22 \mu \mathrm{g} / \mathrm{mL}$ & $4,63 \mu \mathrm{g} / \mathrm{mL}$ \\
\hline I.C. $\mathbf{E C}_{\mathbf{5 0}}(\mathbf{P}<\mathbf{0 , 0 5 )}$ & $22,74-27,97 \mu \mathrm{g} / \mathrm{mL}$ & $0,73-29,35 \mu \mathrm{g} / \mathrm{mL}$ \\
\hline Linearidade (R) & 0,975 & 0,954 \\
\hline
\end{tabular}

Legenda: (I.C.) Intervalo de Confiança, considerando um nível de confiança de 95\%.

Fonte: Elaborado pelo autor lan Jhemes Oliveira Sousa (2017). 
Os resultados obtidos a partir da análise da Luffa operculata frente a meristemas de Allium cepa estão dispostos na Figura que representa a avaliação da exposição dos meristemas em crescimento às concentrações de extrato hidroalcoólico (EBHA) de Luffa operculata durante 72 horas.

Figura 4. Média de crescimento das raízes de Allium cepa dos grupos testados $(n=5)$

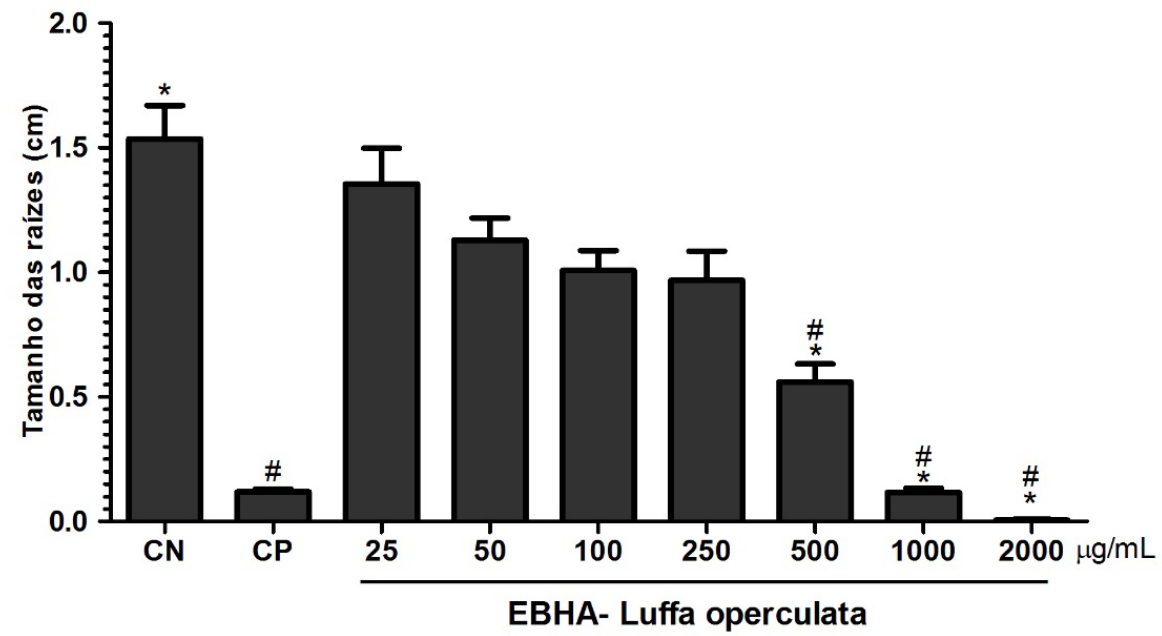

Legenda: Os resultados estão expressos como a média \pm erro padrão da média (E.P.M.) e 5 ensaios independentes (quintuplicata) e significâncias ${ }^{*}$ calculadas para $p<0,05 \%$.

Fonte: Elaborado pelo autor lan Jhemes Oliveira Sousa (2017).

A avaliação empregando os bulbos de Allium cepa visualmente mostra uma inibição do crescimento das raízes na forma concentração dependente, mas a comparação estatística com o controle negativo através do teste de Tukey para um p<0,05 aponta que a inibição do crescimento meristemático obteve significância inicial para a concentração de $500 \mu \mathrm{g} / \mathrm{mL}$ que gerou redução de $64,8 \% \pm 22,56$, e as respectivas inibições de $92,69 \% \pm 5,22$ para $1000 \mu \mathrm{g} / \mathrm{mL}$ e de 100,00\% $\pm 0,01$ para a concentração de $2000 \mu \mathrm{g} / \mathrm{mL}$ considerando um $\mathrm{p}<0,05$.

\section{DISCUSSÃO}

A primeira publicação a utilizar o teste de Artemia salina é datada de 1956, desde então, numerosos artigos utilizam essa abordagem para a avaliação de limites toxicológicos de diversas substâncias, principalmente extratos de plantas ${ }^{(12)}$.

A avaliação de extratos, frações e até mesmo constituintes isolados de plantas são frequentemente avaliadas contra o crustáceo Artemia salina, visto que esse teste é simples, rápido e de baixo custo. As substâncias testadas, quando tem uma $\mathrm{CL}_{50}$ abaixo de $1000 \mu \mathrm{g} / \mathrm{mL}$ são consideradas tóxicas ${ }^{(13)}$. Diversos ensaios de análise toxicológica têm sido ferramenta para análise de diferentes substâncias em Artemia sp ${ }^{(14)}$, como por exemplo, avaliações de toxicidade de lixiviados de aterros sanitários ${ }^{(15)}$; pesticidas organofosforados e carbamatos ${ }^{(16,17)}$; antibióticos ${ }^{(15)}$, entre outros. 
Os estudos de determinação da $\mathrm{CL}_{50}$ em Artemia mostram forte correlação com valores encontrados em camundongos Swiss, o que mostra que esse bioensaio proporciona grandes vantagens na avaliação de toxicidade de derivados de plantas, pois proporcionam informações úteis no prosseguimento de estudos toxicológicos mais avançados, então podendo ser utilizado como ensaio preliminar de químicos tóxicos em mamíferos para determinar a janela de ação tóxica ${ }^{(18-20)}$.

Os resultados encontrados para o extrato hidroalcoólico de Luffa operculata mostram que esta espécie apresenta metabólitos secundários de alta toxicidade, a qual é mostrada no teste que a toxicidade frente ao modelo utilizado que se pronuncia a partir da concentração mais baixa testada, na faixa de 25 partes por milhão (ppm) ou microgramas por mililitro $(\mu \mathrm{g} / \mathrm{mL})$ e havendo uma relação tardia dessa toxicidade visto que em 48 horas de incubação a mortalidade dos náuplios subiu de uma média de $50 \pm 10 \%$ para $90 \%$. Esses resultados anuem com outros dados da literatura que ressaltaram que a Luffa operculata apresenta toxicidade com concentrações baixas quando avaliado frente a morfologia do epitélio mucociliar no palato isolado de rã. ${ }^{(2)}$

A toxicidade do extrato teve início determinado em valores bem baixos, na qual a partir da observação dos dados constata-se que a $\mathrm{Cl}_{50}$ situa-se em um intervalo entre a faixa logarítimica de 5-50 $\mu \mathrm{g} / \mathrm{mL}$ e após a determinação pela análise de regressão através do Gaph Pad Prism determinou-se que a $\mathrm{CL}_{50}$ é aproximadamente 25,22 $\mu \mathrm{g} / \mathrm{mL}$, para o intervalo de 24 horas, o que representa um valor bastante baixo quando comparado com os limites estabelecidos pela literatura, a qual se estabelece que os valores de $\mathrm{CL}_{50}$ menores que $100 \mu \mathrm{g} / \mathrm{ml}$ apresentam alta toxicidade, $\mathrm{CL}_{50}$ entre 100 e $500 \mu \mathrm{g} / \mathrm{ml}$ apresentam toxicidade moderada, $\mathrm{CL}_{50}$ entre de 500 e $1000 \mu \mathrm{g} / \mathrm{mL}$ apresentam fraca toxicidade e $\mathrm{CL}_{50}$ acima de $1000 \mu \mathrm{g} / \mathrm{mL}$ são considerados sem toxicidade ${ }^{(9,21,22)}$, dessa forma, a Luffa operculata mostra uma alta toxicidade visto que sua $\mathrm{CL}_{50}$ é um quarto do valor estabelecido como início do intervalo de alta toxicidade, sendo $<100 \mu \mathrm{g} / \mathrm{mL}$.

$\mathrm{Na}$ análise estatística que permitiu a quantificação da $\mathrm{CL}_{50}$ em Artemia salina, especialmente em 24 horas (o qual consiste no tempo de observação padronizado nos estudos internacionais), foi obtido uma boa linearidade metodológica, visto que, quanto mais se aproximar de 1 , mais precisão o teste tem em relação a dependência entre concentração e a resposta. Nesse panorama, existe também uma relação entre a $\mathrm{CL}_{50}$ teórica e o intervalo de $\mathrm{CL}_{50}$ considerando um $p<0,05$, esse intervalo significa que existem $95 \%$ de chances da "real" $\mathrm{CL}_{50}$ estar situada entre esse intervalo, que para 24 horas, foi de 22,74 a $27,97 \mu \mathrm{g} / \mathrm{mL}$, mostrando uma pequena faixa de erro padrão entre a $\mathrm{CL}_{50}$ obtida e os limites teorizados pela "Sigmoidal Dose-Response".

O método de avaliação de parâmetros toxicológicos por meio do teste de Allium cepa é reconhecido pelo Programa Internacional de Segurança Química (IPCS,OMS) e Programa Ambiental das Nações Unidas (UNEP), podendo oferecer resultados confiáveis e reprodutíveis(23) o que fornece uma maior confiabilidade para as mensurações realizadas por essa metodologia.

Nos resultados obtidos através da metodologia utilizando Allium cepa, as três maiores concentrações foram estatisticamente significativas em comparação ao controle negativo e mostrando-se estatisticamente semelhantes ao controle positivo, que usa concentrações $1000 \mu \mathrm{g} / \mathrm{mL}$ de paracetamol $(1 \mathrm{mg} / \mathrm{mL})$ que tem ação citotóxica/clastogênica bastante evidenciada em meristemas de Allium cepa, ação essa que é atrelada aos metabólitos tóxicos da oxidação do paracetamol, visto que a cebola é rica em oxidases ${ }^{(24)}$.

Dessa forma, postula-se que os compostos de Luffa operculata apresentam citotoxicidade com concentrações maiores que $500 \mu \mathrm{g} / \mathrm{mL}$, pois a inibição do crescimento das raízes deste vegetal se dá pela proliferação contínua e acelerada das células meristemáticas de sua raiz e uma diminuição no crescimento das mesmas pode ser atrelada quase que unicamente à diminuição desta proliferação celular, a qual pode-se atrelar a efeitos adversos causados aos cromossomos, danos ao maquinário de replicação, transcrição, síntese proteica ou citocinese $\mathrm{e}^{(10,25)}$. 


\section{CONCLUSÃO}

Portanto, constata-se a alta toxicidade dos compostos de Luffa operculata, com determinação de $\mathrm{CL}_{50}$ de aproximadamente $25,22 \mu \mathrm{g} / \mathrm{mL}$ e com possível potencial citotóxico com concentrações acima de $500 \mu \mathrm{g} / \mathrm{mL}$, estabelecendo premissas para mais estudos citogenéticos a nível celular para quantificar a ação genotóxica e mutagênica dessa planta que é tanto utilizada indiscriminadamente pela população.

\section{AGRADECIMENTO}

Agradecemos o Centro de Ensino Tecnológico de Teresina e a Universidade Federal do Piauí - UFPI pelo apoio concedido.

\section{REFERÊNCIAS}

1. Caribé RA. Abordagem da atividade biológica do extrato de Luffa operculata Cogn. [dissertação]. Recife (PE): Universidade Federal de Pernanbuco, Programa de Pós-Graduação em Ciências Farmacêuticas; 2008.

2. Menon-Miyake MA, Hilário P, Saldiva N, Lorenzi-Filho G, Ferreira MA, Butugan O, Oliveira RC. Efeitos da Luffa operculata sobre o epitélio do palato de rã: aspectos histológicos. Revista Brasileira de Otorrinolaringologia. 2005; 71(2): 132-138.

3. Pereira RNS, Gonçalves RLG, Sousa IJO, Pinheiro AE, Alves SNT. Análise do potencial antiproliferativo, genotóxico e mutagênico de extrato aquoso da poupa do fruto da Luffa operculata L. em células meristemáticas de Allium cepa (Projeto) In: I Congresso Nacional Acadêmico Profissional de Farmácia no Piauí, II Jornada Acadêmica de Farmácia e I Workshop de projetos e trabalhos em ciências farmacêuticas do Piauí. 2015; Set 1-4; Teresina: União Independente de Farmacêuticos e Acadêmicos de Farmácia do Piauí (Revista Integrada de Ciências Farmacêuticas e Saúde do Piauí. 2015; 02 (1)1-4.

4. Balbino EE, Dias EF. Farmacovigilância: um passo em direção ao uso racional de plantas medicinais e fitoterápicos. Revista Brasileira de Farmacognosia.2010; 20(6): 992-1000.

5. Araújo MGF, Cunha WR, Veneziani RCS. Estudo fitoquímico preliminar e bioensaio toxicológico frente a larvas de Artemia salina Leach. de extrato obtido de frutos de Solanum lycocarpum A. ST.-Hill (Solanaceae). Revista de Ciências Farmacêuticas Básica e Aplicada. 2010; 31(2): 205-209.

6. Goudinho AF, Machado KC, Silva JN, Cavalcante, AAC, Ferreira PMP.Toxicidade do bufadienolídeo Marinobufagina. Anais do $3^{\circ}$ Workshop de projetos e dissertações do Programa de Pós-Graduação em Ciências Farmacêuticas; 2014 Nov. 6-7; Teresina. Piauí: Programa de Pós-Graduação em Ciências Farmacêuticas. 2014; 1(2) 31-34.

7. Bagatini, MD, Fachinetto JM, Silva ACF, Tedesco SB. Cytotoxic effects of infusions (tea) of Solidago microglossa DC. (Asteraceae) on the cell cycle of Allium cepa. Rev. Bras. Farmacogn. 2009; 19(2): 632-636.

8. Tedesco SB, Laughinghouse HD. Bioindicator of genotoxicity: the Allium cepa test. INTECH Open Access Publisher [Internet]. 2016; 137-158: Availabre from: <http://www.intechopen.com/books/environmentalcontamination/ bioindicatorofgenotoxicity-the-alliumcepatest>.

9. Meyer BN, Ferrigni NR, Putnam JE, Jacobsen, LB, Nichol DE, McLaughlin JL. Brine shrimp: A convenient General Bioassay for active plant constituents. Journal of Medicinal Plant Research. 1982; 45(5): 31-34.

10. Fiskejö G. The Allium test as a standard in environmental monitoring. Hereditas. 1985; 102(1): 99-112. 
11. Sturbelle RT, Pinho DS, Restani RG, Oliveira GR, Garcias GL, Martino-Roth MG. Avaliação da atividade mutagênica e antimutagênica da Aloe vera em teste de Allium cepa e teste de micronúcleo em linfócitos humanos binucleados. Rev. bras. Farmacogn. 2010; 20(3): 409-413.

12. Nunes LCC, Galindo AB, Deus ASO, Arcanjo DDR, Randau KP, Xavier HS, Citó AMGL, Rolim-Neto PJ. Variabilidade sazonal dos constituintes da própolis vermelha e bioatividade em Artemia salina. Revista Brasileira de Farmacognosia. 2009; 19(2B): 524-529.

13. Nunes XP, Mesquita RF, Silva DA, Lira DP, Costa VC, Silva MV, et al. Chemical constituents, evaluation of antioxidant and cytotoxic activities of Mimosa paraibana Barneby (Mimosaceae). Rev. Bras. Farmacogn. 2008; 18(1): 718-23.

14. Brix KV, Gerdes RM, Adams WJ, Grosell M. Effects of Copper, Cadmium, and Zinc on the Hatching Success of Brine Shrimp (Artemia fransciscana) .Arch. Environ. Contam. Toxicol. 2006; 51(1): 580-583.

15. Svensson BM, Mathiasson L, Martensson, L. Bergstrom S. Artemia salina as test organism for assessment of acute toxicity of leachate water from landfills. Envoronmental Monitoring and Assessmen. 2005; 102(1): 309-321.

16. Varó I, Amat F, Navarro JC, Barreda M, Pitarch E, Serrano R. Assessment of the efficacy of Artemia sp (Crustacea) cysts chorion as barrier to chlorpyrifos (organophosphorus pesticide) exposure. Effect on hatching and survival. Science of the Environment. 2006; 366(1): 148-153.

17. Barahona MV, Sánchez-Fortún S. Toxicity of carbamates to the brine shrimp Artemia salina and the effect of atropine, BW284c51, iso-OMPA and 2-PAM on carbaryl toxicity. Environmental Pollution. 1999; 104(1): 469-476.

18. Migliore L, Civitareale C, Brambilla G, Di-Delupis GD. Toxicity of several important agricultural antibiotics to Artemia. Wat. Res. 1997; 31(7): 1801-1806.

19. Nunes BS, Carvalho FD, Guilhermino LM Stappen GV. Use of the genus Artemia in ecotoxicity testing. Environ Pollut. 2006; 144(2): 453-456.

20. Arcanjo DD, Albuquerque AC, Melo-Neto B, Santana LC, Medeiros MG, Citó AM. Bioactivity evaluation against Artemia salina Leach of medicinal plants used in Brazilian Northeastern folk medicine. Braz. J. Biol. 2012; 72(1): 505-9.

21. Jose RP, Ramos KP, Gelsano ML, Nuneza OM, Uy MM. Brine Shrimp Lethality Assay of the Ethanolic Extracts of Antidesma ghaesembilla Gaertn. Bull. Env. Pharmacol. Life Sci. 2015; 4(9): 88-92.

22. Nguta JM, Mbaria JM, Gakuya D, Gathumbi PK, Kabasa JD, Kiama S., Biological screening of kenya medicinal plants using Artemia salina L. (Artemiidae). Pharmacologyonline. 2011; 2(1): 458-78.

23. Bagatini MD, Silva ACF, Tedesco SB. Uso do sistema teste de Allium cepa como bioindicador de genotoxicidade de infusões de plantas medicinais. Revista Brasileira de Farmacognosia, João Pessoa. 2007; 17(3): 444-447.

24. Bessems JGM, Gaisser HD, Te-Koppele JM, Van-Bennekon WP, Commandeur JNM, Vermeulen NPE., 3,5-Disubstituted analogues of paracetamol. Synthesis, analgesic activity and cytotoxicity. Chemico-Bilogical Interactions. 1995; 98(1): 237-250.

25. Paula RP, Bueno SSS, Schimitt KFM, Tiago AV, Rossi AAB. Sistema teste de Allium cepa como bioindicador de citotoxicidade e genotixicidade em Aristolochia elegans Mast. Enciclopédia Biosfera. 2015; 11(21): 1749-1756. 